\title{
MODULAR DETECTION SYSTEM FOR SPECIAL NUCLEAR MATERIAL (MODES_SNM)
}

\author{
GEORGIOS CHRISTODOULOU \\ (for the MODES_SNM Collaboration) \\ University of Liverpool, Liverpool, UK \\ Georgios.Christodoulou@liverpool.ac.uk
}

Published 25 February 2014

\begin{abstract}
The MODES_SNM project, funded by the European Community within the scope of the FP7 security theme, explores new techniques for the design and demonstration of novel technologies for the detection of dangerous radioactive materials. Noble gas pressurized detectors are developed and optimized to build a human portable modular detector system to detect and identify illicit SNM. Since masked or shielded SNM is hard to detect, the MODES_SNM detector system will be sensitive to both fast and thermal neutrons and to photons emitted by the SNM. Thus, the project aims to increase the detection sensitivity of shielded SNM, to reduce the false alarm rate and to provide a mobile system to be used by both experts and non-experts in the field of radiation detection. The project now enters into its final phase towards the construction and characterization of a working prototype to be tested under laboratory conditions and in a real world environment.
\end{abstract}

Keywords: MODES; SNM; NORM; gas detector.

\section{Introduction}

The detection of illicit nuclear material is the aim of the Modular Detection System for Special Nuclear Material (MODES_SNM). SNM, like highly enriched uranium and weapons grade plutonium, is difficult to detect, especially when the radioactive source is shielded or masked by natural occurring radioactive material (NORM). The detection of both neutrons and photons emitted by the radioactive source is essential to increase the sensitivity of SNM against natural background and to discriminate the signal of SNM against other sources of radiation. The modularity of the system makes MODES_SNM a perfect tool for radiation detection operation in large crowds, at border controls and near ports or airports.

This is an Open Access article published by World Scientific Publishing Company. It is distributed under the terms of the Creative Commons Attribution 3.0 (CC-BY) License. Further distribution of this work is permitted, provided the original work is properly cited. 


\section{High Pressure Gas Detectors}

MODES_SNM employs high pressure gas detectors, developed by ARKTIS, ${ }^{1}$ to detect both neutron and photons. He-4 is used as an alternative to the nowadays rare and expensive He-3 to detect the neutrons, while xenon is used as the photon detector. The detectors have a cylindrical shape with various dimensions and are internally coated with a wavelength shifter (WLS) and reflector. Each side of the high pressure tube is mounted with a photomultiplier (PMT). The scintillation light produced from an interacting neutron or photon is converted to visible light using the WLS and is then detected by the PMT.

\subsection{Neutron detectors}

High pressure gas ${ }^{4} \mathrm{He}$ tubes at 120-200 bar are used as the fast neutron detectors. With a high cross section probability around $1 \mathrm{MeV}$, approximately where the spectrum of SNM peaks, and high photon rejection efficiency, ${ }^{4} \mathrm{He}$ appears to be a very attractive candidate for ${ }^{3} \mathrm{He}$ replacement. A detailed description of the high pressure ${ }^{4} \mathrm{He}$ detectors with their photon-neutron discrimination power can be found in Ref. 1. Use of a converting material makes the same detector sensitive to thermal neutrons as well.

\subsection{Photon Detectors}

Xenon gas at 20-60 bar is used as the photon detector. With no cryogenics required and some attractive properties (rather dense material, large photoabsorption cross section, and good scintillation and ionization properties) xenon appears as an excellent choice for a photon detector. The energy resolution achieved is 9\% FWHM at $662 \mathrm{keV}$ with a light yield 1.9 p.e./keV at 40 bar pressure. This is comparable to $11.7-14.5 \%$ FWHM achieved at $662 \mathrm{keV}$ with liquid xenon detectors. More details can be found in Ref. 2. Detector optimization and analysis improvements, including optimized reflector and windows WLS coating, result in an improved energy resolution of $6.1 \%$ FWHM at $662 \mathrm{keV}$.

\subsection{Detector optimization}

Currently, the optimization regarding the dimensions and the weight of the fast neutron detectors has been completed (Fig. 1). A fast neutron detector tube has $47 \mathrm{~cm}$ active length and $5.2 \mathrm{~cm}$ diameter. The total length is $100 \mathrm{~cm}$. A significant effort to reduce the weight of the fast neutron detector, including some mechanical changes and replacement of the electronic pressure sensor with a strain gauge system, has been considered. Also, the metallic PMT cases have been replaced with carbon fiber constructed at the University of Liverpool. The external part of the carbon fiber case was coated with a thin nickel layer to provide EMI shielding. The weight of each fast neutron detector is approximately $4.9 \mathrm{~kg}$. The photon detectors are larger in diameter $(11.5 \mathrm{~cm})$ but smaller in length (20 cm active volume and $60 \mathrm{~cm}$ total length) with weights up to $13 \mathrm{~kg}$. 


\section{System Architecture}

The MODES_SNM system contains two blocks. Block A hosts all the detectors: these are eight fast neutron detectors, two slow neutron detectors and two photon detectors. In block B, all the electronics, the batteries and the computer equipment will be placed. As shown in Fig. 2, a wireless communication between the electronics board and the remote computer will be adopted.

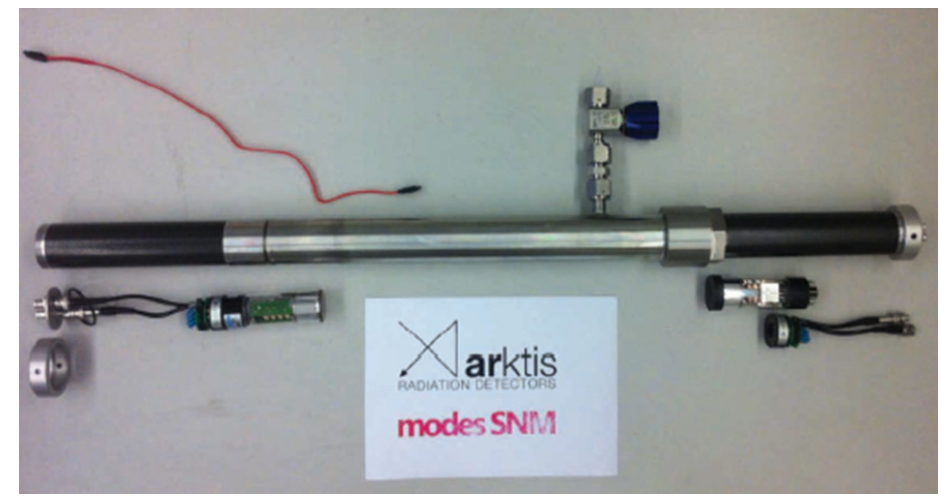

Fig. 1. The high pressure ${ }^{4} \mathrm{He}$ gas fast neutron detector.

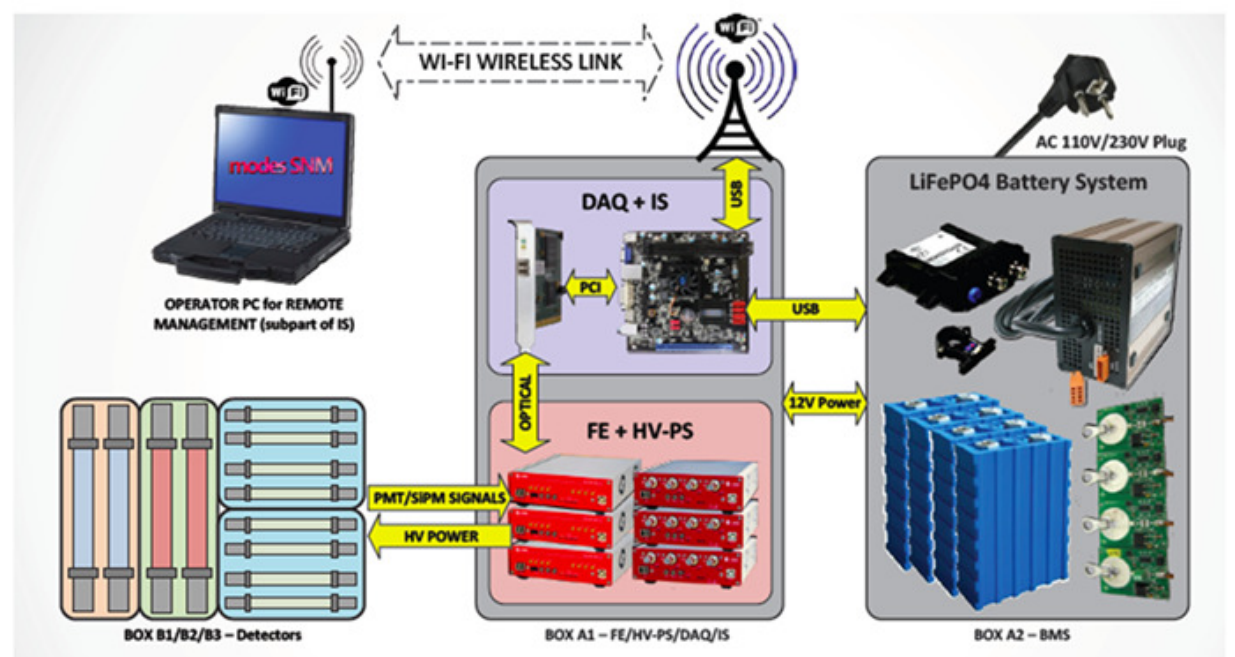

Fig. 2. Architecture of the MODES_SNM system.

\subsection{System integration}

All the equipment (neutron and photon detectors, electronics, batteries and the computer equipment) will be placed in special containers for easy transportation and protection. The containers should be robust and waterproof and should provide EMI shielding and 


\section{G. Christodoulou}

protection from extreme weather conditions. Since this is a mobile system, the containers must also provide stability and safety of the equipment while the whole system is transported. The overall weight of the container itself and the equipment hosted must not exceed $25 \mathrm{~kg}$. Thus, a lightweight material (aluminum or composite materials) is required to construct the containers. All the containers will be placed together at the end to build the two blocks, one for the detectors and the second for all the other equipment. The construction of the containers and the integration of the system are expected to be finished by the end of autumn 2013 .

\subsection{System operation}

The system is expected to operate from inside a van and/or outside. In either case, nonexpert users are expected to be able to operate the system. Thus, the MODES_SNM system is foreseen for two operational modes: one for the non-expert user (Fig. 3) and a second for the expert user (Fig. 4). The non-expert mode will provide simple instructions for the system operation and trigger an alarm in the presence of a radioactive source. The alarm would be different for neutrons and photons. Battery indicator, automatic calibration and live status of the system (warnings, errors and reports printed on the screen) will help the non-expert user to have a smooth operation of the system without the necessity to know all the details of the operation.
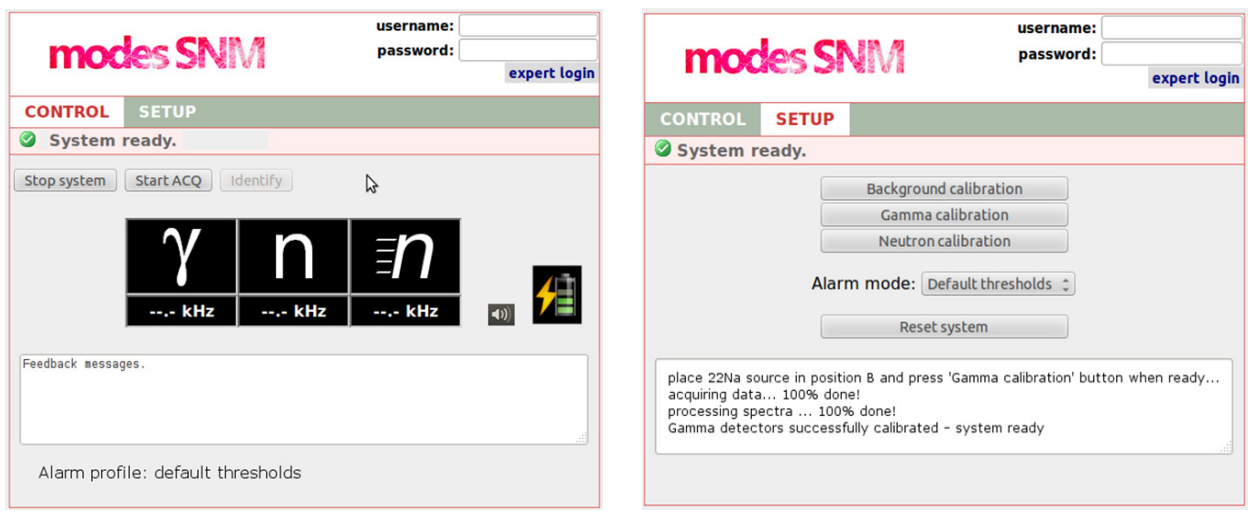

Fig. 3. Demonstration of the non-expert mode for the system operation. 

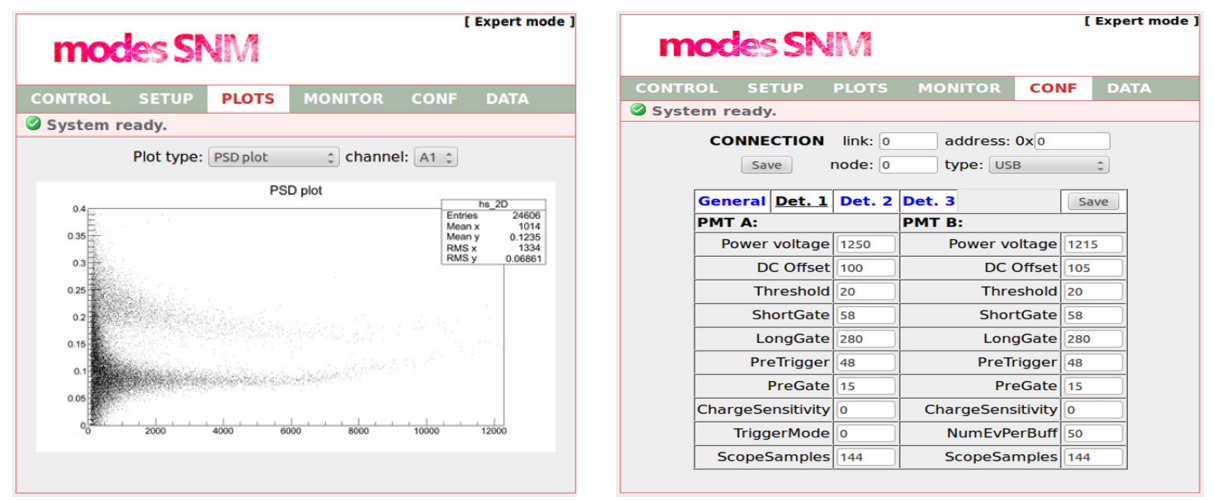

Fig. 4. Demonstration of the expert mode for the system operation.

The password protected expert mode will give the ability, in case it is necessary, for the expert users to run the system. The expert mode will provide more information about system stability, electronics configuration, advance calibration routines, data transfer to other locations and configuration of the Wi-Fi.

\section{Summary}

The construction of the MODES_SNM prototype is now entering its final stage where the electronics and detector optimization are expected to be finished at the end of the 2013 summer. The integration of the system will finish towards the end of the 2013 calendar year. Lab characterization will start soon after, and in the spring of 2014 the field demonstration will take place in three different locations in Europe. The field demonstration will be performed by non-experts.

In the meantime, a lot of other activities are taking place to optimize the performance of the system. These include investigation of possible application of silicon photomultipliers, ${ }^{3}$ detector simulation, application of Compton coincidence technique ${ }^{4}$ to measure the non-proportionality and energy resolution of the pressurized xenon detector, development of source identification algorithms including peak finding analysis of the photon detector, response function analysis, energy window and shape analysis for the neutron detector response.

\section{Acknowledgments}

This work is supported by the European Union through MODES_SNM project (FP7SEC-2011-1, ERC grant agreement $\mathrm{n}^{\mathrm{o}}$ 284842). 
G. Christodoulou

\section{References}

1. Rico Chandra et al., Fast Neutron Detection With Pressurized 4He Scintillation Detectors, in Proc. 2nd International Workshop on Fast Neutron Detectors and Applications, JINST 7 , C03035 (2012).

2. F. Resnati et al., Suitability of High-Pressure Xenon as Scintillator for Gamma Ray Spectroscopy, arXiv:1212.4050 (2012), http://arxiv.org/pdf/1212.4050.pdf.

3. R. Chandra et al., Results from a Noble Gas Scintillation Detector with Solid State Light Readout, this proceedings.

4. B. D. Rooney and J. D. Valentine, IEEE Transactions on Nuclear Science 43(3), 1271 (1996). 